\title{
Ginsenoside Rg1 improves ischemic brain injury by balancing mitochondrial biogenesis and mitophagy
}

\author{
Xuhui Tang ${ }^{*}$, Ningnan Chen and Xiangling Long \\ Department of Emergency, People's Hospital of Jiangxi Province, Nanchang, Jiangxi, 330006, China \\ *For correspondence: Email: xuhtang@163.com
}

Sent for review: 1 August 2017

Revised accepted: 28 September 2017

\begin{abstract}
Purpose: To study the effects of ginsenoside Rg1 on mitochondrial dysfunction induced by ischemic stroke.

Methods: Human neuroblastoma SK-N-SH cells, subjected to oxygen-glucose deprivation (OGD), were divided into six groups: control group, OGD group, 3 OGD + Rg1 groups (6.25, 12.5 and $25 \mu M)$, and $R g 1(25 \mu \mathrm{M})$ group. Apoptosis rate, intracellular production of reactive oxygen species (ROS), and mitochondrial transmembrane potential (MTP) in the OGD cells treated with different concentrations of Rg1 were determined. The mRNA and protein expression levels of mitochondrial biogenesis-related transcription factors and autophagy-related proteins were determined by reat time-polymerase chain reaction (RT-PCR) and Western blotting.

Results: ROS production was significantly increased in OGD SK-N-SH cells $(p<0.01)$, but this was reversed by $R g 1$ treatment ( $p<0.05)$. Rg1-treated cells had significantly higher MTP when compared with OGD cells $(p<0.01)$. Rg1 treatment led to significant increases in $m R N A$ and protein expression levels of PGC1- $\alpha, N R F-1$, and TFAM-1 $(p<0.01)$. Moreover, Rg1 treatment inhibited apoptosis in SK$\mathrm{N}-\mathrm{SH}$ cells, and up-regulated autophagy-related proteins in $t$ neuronal injury model. Treatment with autophagy inhibitors decreased the mitochondrial protective effects exerted by Rg1 in OGD SK-N-SH cells.

Conclusion: Rg1 improves mitochondrial dysfunction by regulating autophagy in mitochondria. Thus, it may offer protection from brain injuries caused by cerebral ischemia.
\end{abstract}

Keywords: Cerebral ischemia, Ginsenoside Rg1, Mitochondrial dysfunction, Mitophagy

Tropical Journal of Pharmaceutical Research is indexed by Science Citation Index (SciSearch), Scopus, International Pharmaceutical Abstract, Chemical Abstracts, Embase, Index Copernicus, EBSCO, African Index Medicus, JournalSeek, Journal Citation Reports/Science Edition, Directory of Open Access Journals (DOAJ), African Journal Online, Bioline International, Open-J-Gate and Pharmacy Abstracts

\section{INTRODUCTION}

Stroke is the third most deadly disease in the world and a major source of disability [1]. Ischemic stroke, also known as cerebral ischemia, accounts for about $65-80 \%$ of strokes, and is caused by lack of cerebral blood flow due to a variety of factors [1,2]. Cell autophagy is extensively involved in various stages of cerebral ischemia [3]. Autophagy is a self-regulating defensive adaptation process in eukaryotic cells $[3,4]$. It is expected to provide raw materials for cell repair and reconstruction to meet the normal turnover and cyclic utilization of cytoplasmic components. Thus, it has an important regulatory role in maintaining the stability of the intracellular environment, cell growth, and development $[3,4]$.

The mitochondrion is an important organelle that produces energy and regulates cell signaling and apoptosis. It is also the main source of reactive oxygen species (ROS) in the cell [5]. The ROS generated from damaged mitochondria may be released into the cytoplasm, where they induce functional and morphological changes, resulting 
in imbalance in intracellular redox metabolism and eventually extensive mitochondrial damage $[5,6]$. Hence, the timely removal of damaged mitochondria is very vital for the normal growth of cells. Mitochondrial autophagy (mitophagy) is a process discovered in recent years, in which the damaged mitochondria are removed by a selective autophagy pathway so as to decrease ROS and promote cell survival by maintaining steady state of intracellular redox status $[7,8]$. If mitochondrial autophagy is inhibited, damaged mitochondria will produce excess ROS and trigger on cell death $[7,8]$. At present, the role of this adaptive metabolic mechanism in the pathogenesis of cerebral ischemia is the subject of research interest to many investigators [9].

Ginsenoside Rg1 is one of the main components of ginseng extract and pharmacologically active compound in clinical applications [10]. It has antioxidant and anti-aging properties, and has been used to alleviate nerve function impairment, and for the treatment of cerebrovascular diseases [10]. Recently studies reported that ginsenosides inhibit mitochondrial dysfunction via regulation of imbalances in mitochondrial dynamics [11,12].

This study was aimed at investigating the effect of Rg1 on mitochondrial dysfunction due to cerebral ischemia.

\section{EXPERIMENTAL}

\section{Cell culture and Rg1 preparation}

Human neuroblastoma SK-N-SH cells were obtained from Sigma-Aldrich (USA). The cells were cultured at $37^{\circ} \mathrm{C}$ in Dulbecco's Modified Eagle's Medium (DMEM; Invitrogen, USA) containing $2 \mathrm{mM}$ glutamine and $10 \%$ Fetal Bovine Serum (FBS) in a humidified environment containing $5 \% \mathrm{CO}_{2}$. Rg1 solution (100 mM; Meilunbio, China) was prepared in dimethylsulfoxide and diluted to the indicated final concentrations with DMEM medium before treatment.

\section{Oxygen-glucose deprivation (OGD)}

In order to subject SK-N-SH cells to OGD, the cell culture media was replaced by glucose-free DMEM (Invitrogen, USA). Then, the cells were placed in an incubator containing $95 \% \mathrm{~N}_{2}$ and 5 $\% \mathrm{CO}_{2}$ for $4 \mathrm{~min}$. The culture chamber was then sealed and the cells were incubated for $4 \mathrm{~h}$ at $37^{\circ} \mathrm{C}$. Cells in the control group were cultured in glucose-containing DMEM in a normoxic condition for $4 \mathrm{~h}$. The SK-N-SH cells were pretreated either with vehicle or Rg1 (6.25, 12.5, and $25 \mu \mathrm{M}$ ) for $24 \mathrm{~h}$ and then subjected to OGD. Cells were divided into six groups: cells treated with vehicle (control group), cells treated with OGD (OGD group), cells treated with OGD and different concentrations of $\mathrm{Rg} 1$ (6.25, 12.5 and $25 \mu \mathrm{M})$; and cells treated with $25 \mu \mathrm{M} \mathrm{Rg} 1$.

\section{Apoptosis assay}

Apoptosis in OGD-treated neuronal injury model exposed to different concentrations of Rg1 was determined using annexin $\mathrm{V}$ assay (BD, USA) according to the manufacturer's protocol. The apoptotic cells were identified by flow cytometry.

\section{Measurement of ROS}

Intracellular production of ROS was determined using a DCFDA Cellular ROS detection assay kit (Abcam, UK). The fluorescence intensity of carboxy- $\mathrm{H}_{2}$ DCFDA (excited at $488 \mathrm{~nm}$ ) was measured using a FACSCanto II cytometer (BD, USA), to determine the ROS level in each group.

\section{Measurement of mitochondrial transmembrane potential (MTP)}

A TMRE MTP assay kit (Abcam, UK) was used to determine MTP. The cells were incubated in a medium containing $10 \mu \mathrm{M}$ tetramethylrhodamine (TMRE, ethyl ester) for $20 \mathrm{~min}$ at $37^{\circ} \mathrm{C}$ (in this assay, mitochondria with decreased membrane potential are not stained by TMRE). Then, the cells were transferred to a clear 96-well plate, and the TMRE staining was measured with a microplate spectrophotometer (excitation at 549 $\mathrm{nm}$ and emission at $575 \mathrm{~nm}$ ).

\section{Western blot analysis}

Cells were harvested and washed with cold phosphate buffer solution for twice. Then, the cells were lysed in radioimmunoprecipitation assay (RIPA) buffer (Sigma-Aldrich, USA). The total protein concentrations were determined using Pierce ${ }^{\mathrm{TM}}$ BCA protein assay kit (Thermo Fisher Scientific, USA). The proteins $(25 \mu \mathrm{g})$ were subjected to $10 \%$ SDS-PAGE and then electro-transferred onto a nitrocellulose membrane. The transferred proteins were then probed with primary antibodies against PGC1- $\alpha$, NRF1, Tfam, LC3B II, p62, and beclin-1 (Abcam, USA). $\beta$-actin was loaded as an internal reference. Then the membranes were further probed with goat anti-rabbit IgG-HRP secondary antibody (1:2000; Abcam, USA). Bands were developed using Pierce ${ }^{\mathrm{TM}}$ electrochemiluminescence (ECL) substrate (Thermo Fisher Scientific, USA). 


\section{RT-PCR}

RNA was extracted by with RNeasy Plus Micro Kit (QIAGEN, USA), according to the manufacturer's instructions. Then, the reverse transcription of the extracted RNA was performed with the SuperScript $\AA$ IV First-Strand Synthesis System (Invitrogen, USA). RT-PCR was performed in Applied Biosystems 7500 Real Time PCR System (Applied Biosystems, USA), using $20 \mathrm{ng}$ template in $25 \mu \mathrm{L}$ reaction volume with $2 \times$ Power SYBR $\AA$ Green PCR Master Mix (Invitrogen, USA) and gene specific primer pairs for PGC1- a, NRF1, Tfam, LC3B II, p62, beclin-1, and $\beta$-actin. The PCR program setting was as follows: $95^{\circ} \mathrm{C}, 10 \mathrm{~min} ; 45$ cycles of $95^{\circ} \mathrm{C}, 15 \mathrm{~s}$, and $60^{\circ} \mathrm{C}, 1 \mathrm{~min}$. The gene expression levels for all samples were normalized to $\beta$-actin mRNA expression using the comparative $\mathrm{Ct}$ method.

\section{Statistical analysis}

All the experiments were done in triplicates and the SD was calculated. Analysis of variance (ANOVA) and multiple comparisons using Dunnett's test were applied to compare the differences between groups. $P<0.05, p<0.01$, or $p<0.001$ was considered statistically significant.

\section{RESULTS}

\section{Rg1 treatment inhibited OGD-induced apoptosis in SK-N-SH cells}

An in vitro neuronal injury model was successfully established by subjecting the human neuroblastoma SK-N-SH cells to OGD. As shown in Figures $1 \mathrm{~A}$ and Figure 1B, OGD treatment alone significantly increased the apoptotic rate of SK-N-SH cells $(p<0.01$, when compared with the control group. However, this increase in apoptosis was significantly and dosedependently inhibited by pre-treatment with $\mathrm{Rg} 1$ $(p<0.05)$.
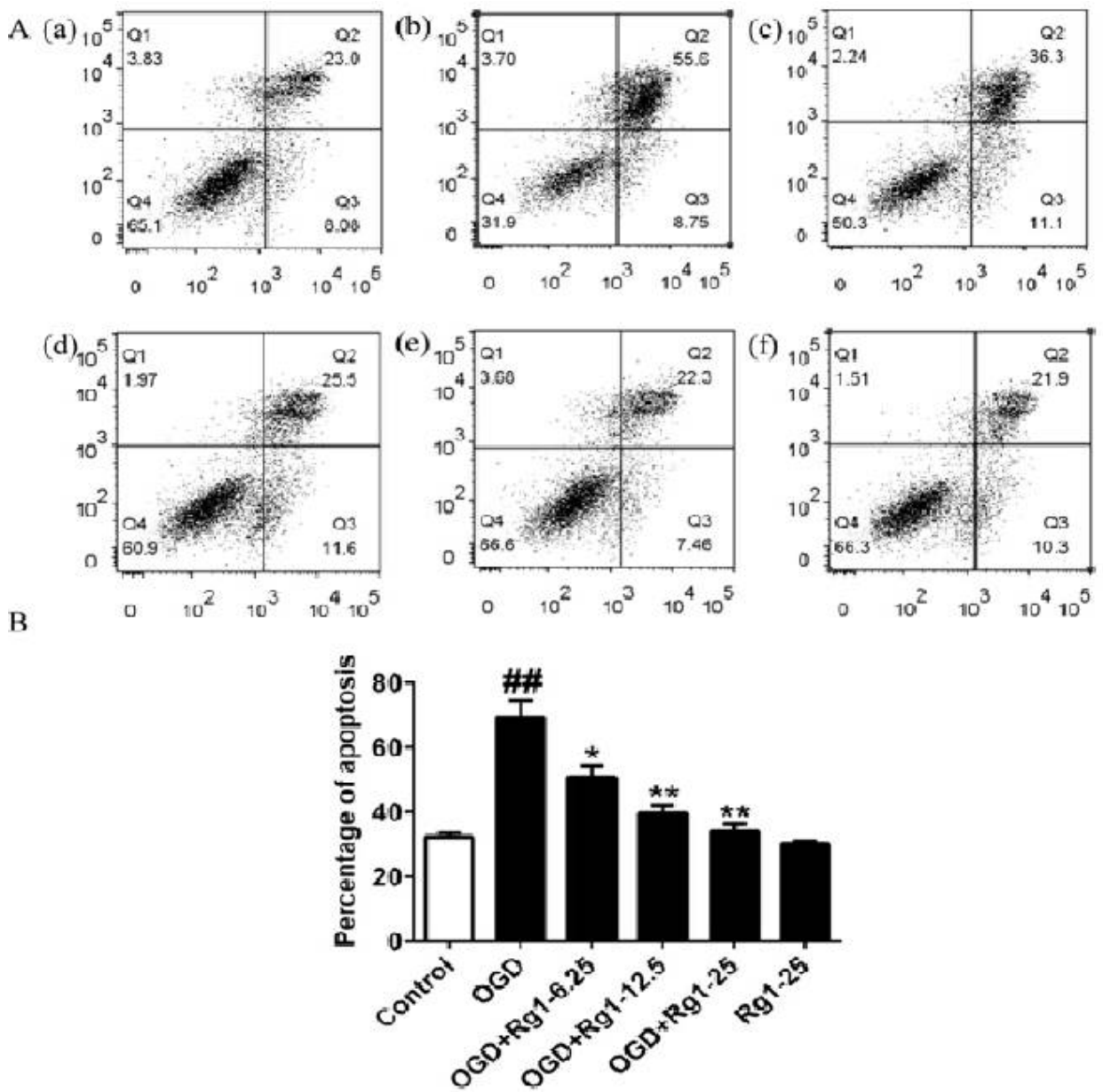

Figure 1: Inhibition of OGD-induced apoptosis in SK-N-SH cells by Rg1 treatment (A) Representative dot plots of apoptosis rate measured by flow cytometry. (a) control group; (b) OGD group; (c) OGD + $6.25 \mu \mathrm{M}$ Rg1 group; (d) OGD + $12.5 \mu \mathrm{M}$ Rg1 group; (e) OGD + $25 \mu \mathrm{M}$ Rg1 group; (f) $25 \mu \mathrm{M}$ Rg1 group. (B) Quantitative analysis of percentage of apoptotic death. \#\# $p<0.01$, versus the control group; ${ }^{*} p<0.05,{ }^{* *} p<0.01$ versus the OGD group Rg1 treatment alleviated OGD-induced ROS

production in SK-N-SH cells 
In order to study the effect of Rg1 on ROS production in neuronal injury model, a DCFDA cellular ROS detection assay was applied. The results showed that ROS production was significantly increased in SK-N-SH cells subjected to OGD $(p<0.01$ relative to the control group; Figure 2). The $\mathrm{Rg} 1$ treatment also significantly attenuated ROS production in the neuronal injury model when compared to the OGD group $(p<0.05$ at 12.5 and $25 \mu \mathrm{M}$ Rg1 treatments).

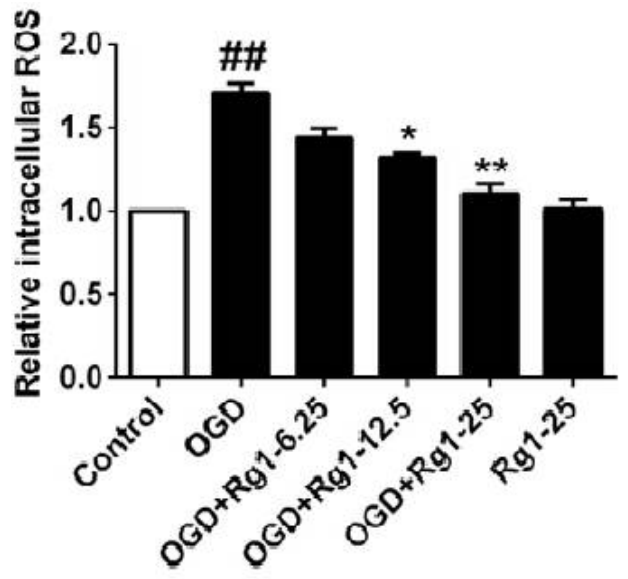

Figure 2: Effect of $\mathrm{Rg} 1$ on intracellular ROS production in OGD SK-N-SH cells. \#\# $p<0.01$, versus the control group; * $p<0.05$, ${ }^{* *} p<0.01$, versus the OGD group

\section{Effect of Rg1 treatment on MTP in SK-N-SH cells with OGD}

Results showed that OGD treatment significantly decreased MTP in the SK-N-SH cells, when compared with the control $(p<0.01$, Figure 3$)$. However, treatment with Rg1 reversed the OGDinduced decreases in MTP by bringing about significant and concentration-dependent increases in MTP, relative to the OGD $(p<0.01$ at 12.5 and $25 \mu \mathrm{M}$ of $\mathrm{Rg} 1$ ).

\section{Effect of Rg1 treatment on mitochondrial biogenesis in neuronal injury model}

The effect of Rg1 on mitochondrial biogenesis in neuronal injury model was further determined. SK-N-SH cells underwent OGD showed a slightly increased pattern of expressions of the mitochondrial biogenesis related transcription factors PGC1- $\alpha$, NRF-1, and TFAM-1, as determined by RT-PCR (Figure 4B), as well as increases in protein levels detected by Western blotting (Fig. 4A). Moreover, Rg1 (25 $\mu \mathrm{M})$ treatment on SK-N-SH cells further up-regulated the mitochondrial biogenesis-associated transcription factors, as demonstrated by RT-
PCR and Western blotting in the neuronal injury model ( $p<0.01$, versus the OGD group).

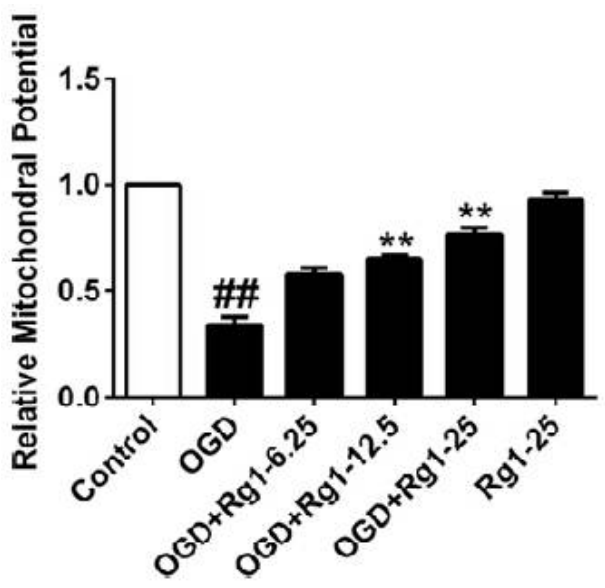

Figure 3: Effect of $\mathrm{Rg} 1$ on MTP in SK-N-SH cells subjected to OGD. \#\#p $<0.01$, versus the control group; ${ }^{*} p<0.05,{ }^{* *} p<0.01$, versus the OGD group

\section{Effect of Rg1 on expressions of autophagy proteins}

The effect of $\mathrm{Rg} 1$ on the expressions of autophagy-related proteins (LC3B-II, p62, and Beclin-1) was studied in vitro. The results showed that the expression levels of LC3B-II and Beclin-1 were significantly up-regulated in Rg1treated OGD SK-N-SH cells, relative to the control group and the OGD-only group $(p<0.01$ and $p<0.05$, respectively; Figures $5 \mathrm{~A}$ and Figure 5B). However, Rg1 treatment had no appreciable effect on p62 levels. In addition, Rg1 treatment failed to reverse the OGD-induced decrease in MTP when the autophagy inhibitors 3-methyladenine (3MA) and wortmannin (wort) were applied (Figure $5 \mathrm{C}, p<0.01$, versus the $\mathrm{OGD}+\mathrm{Rg} 1$ group). These results indicate that Rg1 exerts mitochondrial protective effect on OGD-treated SK-N-SH cells via activation of autophagy.

\section{DISCUSSION}

Ischemic stroke is a serious threat to human health, and easily leads to ischemic neuronal damage, cognitive and physical dysfunction and high mortality $[1,13]$. Currently, ischemic stroke is effectively treated by injecting recombinant tissue plasminogen activator for thrombolysis, but due to the limitations of time window (within 4-5 h after incidence), only few ischemic stroke patients are lucky to receive this therapy [14]. Therefore, it has become necessary to explore new therapeutic targets and drugs for the treatment of stroke. 
A

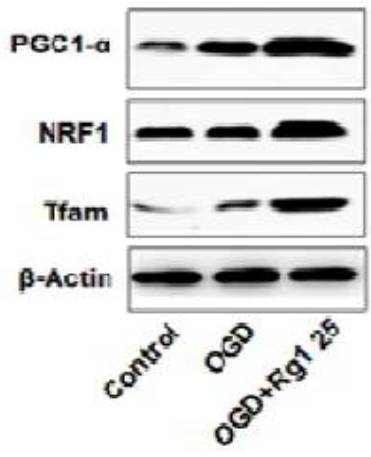

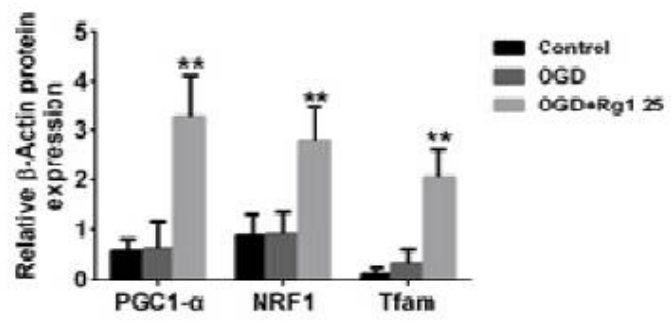

B
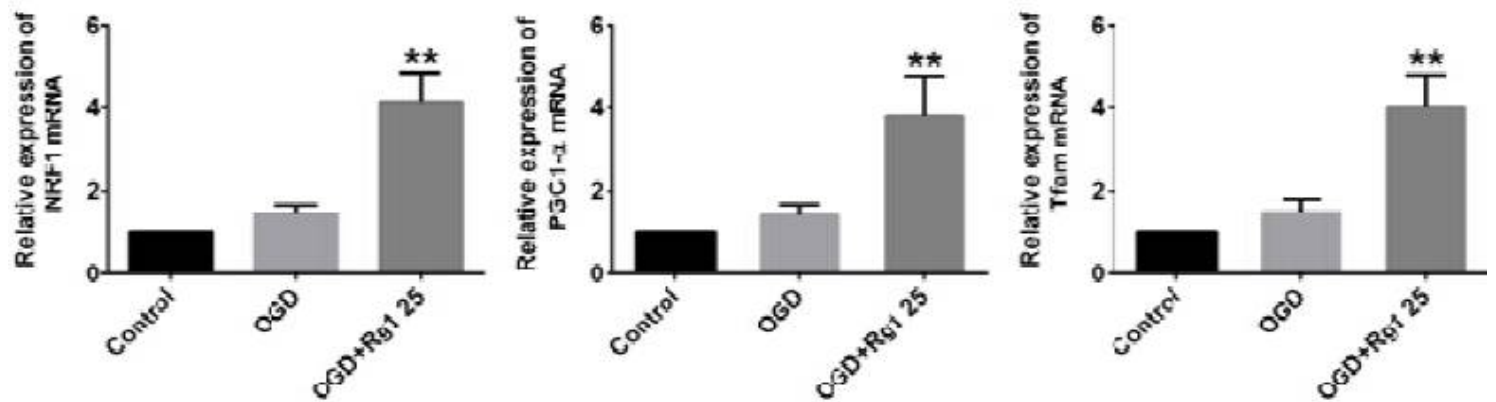

Figure 4: Effect of Rg1 treatment on expressions of mitochondrial biogenesis in neuronal injury model. Western blot (A) and RT-PCR (B) analysis of the expression levels of mitochondrial biogenesis- related transcription factors PGC1- $\alpha$, NRF-1, and Tfam-1 in OGD-treated SK-N-SH cells. ${ }^{*} p<0.01$, versus the OGD group.

A

B
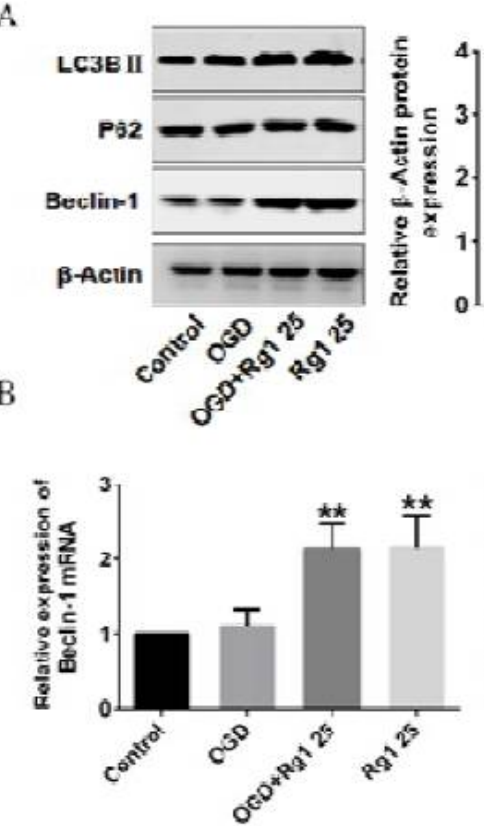
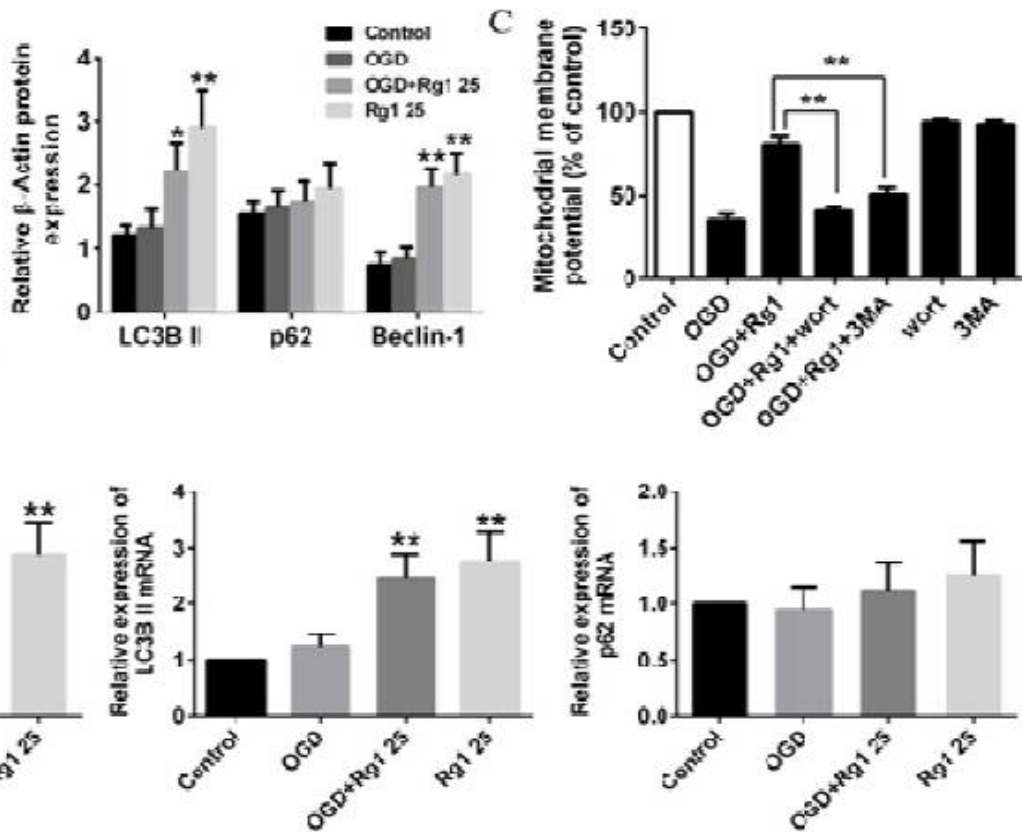

Figure 5: Protective effect of $\mathrm{Rg} 1$ on mitochondrial function in OGD-treated SK-N-SH cells via autophagy activation. Western blot (A) and RT-PCR (B) analyses of the expression levels of autophagy-related proteins LC3B II, p62, and Beclin-1. (C) Rg1 treatment failed to reverse the OGD-induced decrease in MTP when autophagy inhibitors were applied; ${ }^{*} p<0.05,{ }^{*} p<0.01$, compared to the OGD group or as indicated in the figure.

Energy metabolic disorder is an early pathologic response to cerebral ischemic injury [2]. The mitochondrion is the most important energy organelle of cells, providing most of the energy needed for cellular activities, including metabolism and excitatory transmission of cerebral neuronal cells [8,9]. During ischemia and hypoxia, the mitochondria produce a large number of ROS. This results in lipid peroxidation of cell and organelle membranes, loss of membrane integrity and permeability, and ultimately cell death $[5,6,9,13]$.

Excessive ROS can lead to sustained opening of mitochondrial permeability transition pore (MPTP) and membrane damage, impaired 
mitochondrial $\mathrm{Ca}^{2+}$ recycling, decline in MTP, and activation of mitochondrial apoptotic pathway $[5,6,9,13]$.

Ginseng is a drug commonly used for the treatment of cardiovascular and cerebrovascular diseases. It contains ginsenoside, ginseng polysaccharide, proteins, polypeptides, and amino acids, but ginsenoside is the most important active ingredient [10]. In the present study, Rg1 inhibited apoptosis and ROS production in SK-N-SH cells subjected to OGD. In addition, Rg1 treatment increased MTP, when compared with cells subjected to OGD only. Changes in MTP result in impairment of ATP production, which leads to further production of ROS, damage of mitochondrial membrane or even damage of the entire cell $[15,16]$. Therefore, Rg1 may alleviate mitochondrial dysfunction induced by OGD via promoting ATP production in this neuronal injury model.

In vitro ischemia-reperfusion experiments of mouse cortical neurons showed that cortical neurons had decreased mtDNA after ischemia reperfusion, with decreased mitochondrial mass and lowered generative capacity $[17,18]$. Animal ischemia-reperfusion model studies have shown that promotion of mitochondrial production, maintenance of stability of mitochondrial function and increasing energy supply can reduce the incidence of cerebral infarction and neuronal apoptosis $[17,19]$. Therefore, the effect of Rg1 on the mitochondrial biogenesis-associated transcription factors was studied in the SK-N-SH cells subjected to OGD. It was found that Rg1 treatments consistently promoted mitochondrial biogenesis.

Mitochondrial autophagy can delay and decrease the accumulation of mtDNA mutations [7]. When the body is exposed to adverse external stimuli, selective mitochondrial autophagy may occur [7]. Mitochondrial damage may lead to the loss of function and thus trigger mitochondrial autophagy, which is an important way of removing damaged mitochondria to prevent ROS accumulation [7]. In recent years, many experiments have shown that cerebral ischemia is closely related to mitochondrial autophagy. A study by Carloni et al showed that improving mitochondrial autophagy can perfect cell morphology and reduce tissue necrosis, thus enhancing neuro-protection [20]. The present study found that $\mathrm{Rg} 1$ can un-regulate the autophagy-related proteins and activate autophagy in the neuronal injury model studied. The mitochondrial protective function exerted by Rg1 decreased after application of autophagy inhibitors in vitro. Cell autophagy has destructive effect, so the inhibition of autophagy can protect neurons [21]. Therefore, autophagy may be a double-edged sword: under mild physiological stress, the activation of mitochondrial autophagy has a protective effect, while in severe ischemia and hypoxia, it may be excessively activated, leading to neuronal damage and necrosis [21]. Therefore, the regulation of autophagy and inhibition of signaling pathways of various damaged mitochondria may be new targets of neuroprotective agents.

\section{CONCLUSION}

The results obtained in this study demonstrate that $\mathrm{Rg} 1$ can relieve mitochondrial dysfunction by regulating autophagy in mitochondria. Thus, it has the potential to be developed as a therapeutic agent for brain injuries caused by cerebral ischemia.

\section{DECLARATIONS}

\section{Acknowledgement}

None.

\section{Conflict of Interest}

No conflict of interest associated with this work.

\section{Contribution of Authors}

The authors declare that this work was done by the authors named in this article and all liabilities pertaining to claims relating to the content of this article will be borne by them.

\section{Open Access}

This is an Open Access article that uses a funding model which does not charge readers or their institutions for access and distributed under the terms of the Creative Commons Attribution License (http://creativecommons.org/licenses/by/ 4.0) and the Budapest Open Access Initiative (http://www.budapestopenaccessinitiative.org/rea d), which permit unrestricted use, distribution, and reproduction in any medium, provided the original work is properly credited.

\section{REFERENCES}

1. Krishnamurthi RV, Moran AE, Feigin VL, Barker-Collo $S$, Norrving $B$, Mensah GA, Taylor $S$, Naghavi $M$, Forouzanfar MH, Nguyen $\mathrm{G}$, et al. Stroke Prevalence, Mortality and Disability-Adjusted Life Years in Adults Aged 20-64 Years in 1990-2013: Data from the Global 
Burden of Disease 2013 Study. Neuroepidemiol 2015; 45: 190-202.

2. Samary CS, Pelosi P, Silva PL, Rocco PRM. Immunomodulation after ischemic stroke: potential mechanisms and implications for therapy. Crit Care 2016; 20: 391.

3. Sheng $R$, Qin ZH. The divergent roles of autophagy in ischemia and preconditioning. Acta Pharmacol Sin 2015; 36: 411-420.

4. Nixon RA, Yang DS. Autophagy and Neuronal Cell Death in Neurological Disorders. Csh Perspect Biol 2012; 4:

5. Tait SWG, Green DR. Mitochondria and cell signalling. J Cell Sci 2012; 125: 807-815.

6. Bhattacharyya A, Chattopadhyay R, Mitra S, Crowe SE. Oxidative stress: an essential factor in the pathogenesis of gastrointestinal mucosal diseases. Physiol Rev 2014; 94: 329-354.

7. Ding $W X$, Yin XM. Mitophagy: mechanisms, pathophysiological roles, and analysis. Biol Chem 2012; 393: 547-564.

8. Lionaki E, Markaki M, Palikaras K, Tavernarakis N. Mitochondria, autophagy and age-associated neurodegenerative diseases: New insights into a complex interplay. Biochim Biophys Acta 2015; 1847 : 1412-1423.

9. Tang YC, Tian HX, Yi T, Chen HB. The critical roles of mitophagy in cerebral ischemia. Protein Cell 2016; 7: 699-713.

10. Lee $\mathrm{CH}, \mathrm{Kim} \mathrm{JH}$. A review on the medicinal potentials of ginseng and ginsenosides on cardiovascular diseases. $J$ Ginseng Res 2014; 38: 161-166.

11. Dong GT, Chen TB, Ren XC, Zhang ZF, Huang WX, Liu L, Luo $P$, Zhou $H$. Rg1 prevents myocardial hypoxia/reoxygenation injury by regulating mitochondrial dynamics imbalance via modulation of glutamate dehydrogenase and mitofusin 2. Mitochondrion 2016; 26: 7-18.

12. Moon JH, Lee JH, Lee YJ, Park SY. Autophagy flux induced by ginsenoside-Rg3 attenuates human prion protein-mediated neurotoxicity and mitochondrial dysfunction. Oncotarget 2016; 7: 85697-85708.

13. Baburamani AA, Ek CJ, Walker DW, Castillo-Melendez M. Vulnerability of the developing brain to hypoxicischemic damage: contribution of the cerebral vasculature to injury and repair? Front Physiol 2012; 3: 424.

14. Gravanis I, Tsirka SE. Tissue-type plasminogen activator as a therapeutic target in stroke. Expert Opin Ther Tar 2008; 12: 159-170.

15. Quoilin C, Mouithys-Mickalad A, Lecart S, FontaineAupart MP, Hoebeke M. Evidence of oxidative stress and mitochondrial respiratory chain dysfunction in an in vitro model of sepsis-induced kidney injury. BbaBioenergetics 2014; 1837: 1790-1800.

16. Singer $M$. The role of mitochondrial dysfunction in sepsisinduced multi-organ failure. Virulence 2014; 5: 66-72.

17. Li J, Ma X, Yu W, Lou Z, Mu D, Wang Y, Shen B, Qi S. Reperfusion promotes mitochondrial dysfunction following focal cerebral ischemia in rats. PLoS One 2012; 7: e46498.

18. Wang XW, Ding SH. Pre-B-cell colony-enhancing factor as a target for protecting against apoptotic neuronal death and mitochondrial damage in ischemia. Neural Regen Res 2016; 11: 1914-1915.

19. Woodruff TM, Thundyil J, Tang SC, Sobey CG, Taylor SM, Arumugam TV. Pathophysiology, treatment, and animal and cellular models of human ischemic stroke. Mol Neurodegener 2011; 6: 11

20. Carloni S, Buonocore G, Longini M, Proietti F, Balduini $W$. Inhibition of rapamycin-induced autophagy causes necrotic cell death associated with Bax/Bad mitochondrial translocation. Neuroscience 2012; 203: 160-169.

21. Chen W, Sun Y, Liu K, Sun X. Autophagy: a doubleedged sword for neuronal survival after cerebral ischemia. Neural Regen Res 2014; 9: 1210-1216. 